\title{
Looking forward to 2013!
}

\author{
Pierre Dutilleul • Ashis SenGupta
}

Published online: 8 November 2012

(C) Springer Science+Business Media New York 2012

As the end of the current year is getting closer, we thought it was a good idea to look back at what 2012 has been for EEST and even more importantly and excitingly, to look ahead and prepare for 2013 !

Including this issue, 2012 has seen the publication of many very interesting EEST articles with thoughtful and innovative content. Even though no special issue or conference proceedings have been published this year, the door remains wide open for such projects; timing is important and project coordinators are encouraged to contact us as soon as possible, before the conference takes place in the case of proceedings.

More technical or procedural issues concern the submission and review of manuscripts. Occasionally, we receive queries concerning the maximum length of the manuscripts submitted to EEST, and our response is that length (including figures and tables) should not be a problem as long as it is justified. Starting in January 2013, a new category of EEST submissions, called "review articles", will be available on Editorial Manager. A very small number of review articles have been published in EEST since its creation, and this new category of submissions is our response to an increasing demand for such publications. We also take this opportunity to warmly send out invitations for EEST submissions on particularly important topics because of their timeliness or impact, which would include a discussion by experts in the field and a rejoinder by the authors. All of this is intended to maintain and even increase an impact factor that has been 1.31 in 2011. The acceptance rate fluctuating around 25-30\%, a quick but efficient and fair turn-over will continue to be the goal. To help EEST

\section{P. Dutilleul (凶)}

McGill University, Montreal, QC, Canada

e-mail: pierre.dutilleul@mcgill.ca

\section{A. SenGupta}

Indian Statistical Institute, Kolkata, India

e-mail: amsseng@gmail.com 
associate editors in this task, authors are now invited to suggest experts as potential reviewers for their manuscript and provide their coordinates with a brief justification, with the understanding that these experts have no personal or direct relationship with them in order to avoid conflicts of interest. This optional request of suggesting potential reviewers has been offered for the first time on Editorial Manager this fall, but might well become a mandatory request later.

Concerning the research thematics that are welcome for EEST contributions, it is important to bear in mind first of all that the EEST abbreviation means Environmental and Ecological Statistics. Accordingly, we encourage and invite submissions with sufficient originality in the development or the application of statistical methods in ecology and the environmental sciences. The spectrum of methods includes spatial and temporal statistics, multivariate and multi-scale analyses, environmetrics, sampling and inference. Themes include climate change, air-soil-water pollution, epidemiology, natural catastrophes, and plant and animal ecology. Biological systems being of increasing complexity and heterogeneity in multiple dimensions and directions, it is more and more frequent, without being a prerequisite, that several methods and more than one theme be involved in an EEST article. Eventually, the novel statistical methods developed and the new results obtained with them should allow policymakers to take better decisions in environmental and ecological issues, based on the principle that better information leads to better judgment.

As the Year 2012 is closing on a 'Happy End' for EEST, we wish everyone, authors, editor and publisher staffs a very enjoyable and promising beginning of 2013, with many significant research publications in the journal later in the year! 